\title{
Effect of the surface charge of silica nanoparticles on oil recovery: wettability alteration of sandstone cores and imbibition experiments
}

\author{
Merlis P. Alvarez-Berrios ${ }^{1} \cdot$ Lisa M. Aponte-Reyes ${ }^{1} \cdot$ Lissette M. Aponte-Cruz $^{1} \cdot$ Paula Loman-Cortes $^{2}$. \\ Juan L. Vivero-Escoto ${ }^{2,3}$
}

Received: 22 January 2018 / Accepted: 31 July 2018 / Published online: 6 August 2018

(c) The Author(s) 2018

\begin{abstract}
Recently, inorganic nanomaterials have emerged as promising wettability modifiers to improve oil recovery. Among them, silica nanoparticles (SNPs) have gained a privileged place due to their outstanding properties. Several studies have reported the effectiveness of SNPs on this particular application. However, there is an increasing interest of understanding the parameters that may play an important role on oil recovery using nanofluids. The impact of particle size, particle concentration and types of nanoparticles on oil recovery have been reported. Nevertheless, to our knowledge, the influence of the surface charge has not been investigated. In this work, the effect of the surface charge of SNPs on oil recovery was studied. Silica nanoparticles with different charge profiles (negative, positive and slightly negative) were synthesized and characterized using transmission electron microscopy, FT-IR, dynamic light scattering and $\zeta$-potential. Negatively charged nanoparticles (Neg-SNPs; $-33.45 \pm 2.75$ ) were obtained by following the Stöber process. The silanol groups present on the surface of the Neg-SNPs are responsible for the negative surface charge. Positively charged nanoparticles (AP-SNPs; $+42.25 \pm 1.9$ ) and slightly negatively charged nanoparticles (MeO-PEG-SNPs; $-12.20 \pm 0.42$ ) were obtained by grafting (3-aminopropyl) triethoxysilane and methoxy polyethylene glycol $\left(\mathrm{MeO}-\mathrm{PEG}_{2 \mathrm{~K}}\right)$ onto the surface of Neg-SNPs, respectively. The impact of silica materials with different charge profiles on wettability alteration and oil displacement at different concentrations (100 or $200 \mathrm{mg} / \mathrm{L}$ ) was evaluated by contact angle estimation and spontaneous imbibition experiments. The results demonstrated that the surface charge of SNPs transformed the wettability of the sandstone cores and impacted oil recovery in a different extent. While MeO-PEG-SNPs showed the best performance at a low concentration, Neg-SNPs were the most effective in changing the rock wettability and removing oil from sandstone cores at a higher concentration. Overall, our results not only allowed to identify the impact of surface charge on oil recovery but also the effect of SNPs concentration on the suitability of the treatment for enhancing the oil recovery process.
\end{abstract}

Keywords Silica nanoparticles $\cdot$ Rock wettability $\cdot$ Oil recovery and contact angle

\section{Introduction}

Electronic supplementary material The online version of this article (https://doi.org/10.1007/s40089-018-0243-5) contains supplementary material, which is available to authorized users.

Merlis P. Alvarez-Berrios mpalvare@ponce.inter.edu

1 Department of Science and Technology, Inter American University of Puerto Rico, Ponce Campus, Ponce, PR 00716, USA

2 Department of Chemistry, The University of North Carolina at Charlotte, Charlotte, NC 28223, USA

3 The Center for Biomedical Engineering and Science, The University of North Carolina at Charlotte, Charlotte, NC 28223, USA
Because wettability is one of the most important properties controlling oil recovery $[2,7,26]$, the efficiency of this process has been improved using wettability modifiers such as surfactants and polymers [6, 29]. The utilization of these agents can induce changes in the wettability of the rock surface from an oil-wet to a water-wet state, which affects the flow behavior of water and oil in porous media [6]. Nevertheless, the use of polymers and surfactants is limited because their mechanical and shear properties are significantly affected at the high temperatures and salinity present in oil reservoirs [6, 17]. Therefore, it is critical to find novel approaches to enhance the oil recovery process. For 
decades, nanomaterials have attracted considerable interest due to their novel properties in a wide variety of scientific areas $[5,12,28,34]$. Recently, the utilization of nanoparticles (NPs) for oil recovery has been proposed and evaluated [19, 24, 25, 34].

Different studies have reported that NPs can improve the wettability of the reservoir rock and they are more stable than surfactants and polymers at the conditions found in porous media [6, 34]. Emerging inorganic nanoparticles such as iron oxide, titanium oxide, aluminum oxide and silica have been used for this application $[9,14,16,21]$. A comparative study using different types of nanofluids demonstrated that silica nanoparticles (SNPs) had one of the best performances for enhancing oil recovery [19]. This is one of the reasons why this type of nanoparticles has attracted wide spread interest for oil displacement from porous media. Additionally, SNPs involve low cost of fabrication, stable structure and their surface can be easily modified by known chemical methods $[23,27]$. There are several studies demonstrating their effectiveness in the wettability alteration of reservoir rocks [1, 10, 18, 25]. For example, Ju et al. studied the change in the wettability of the sandstone surface using lipophobic and hydrophilic polysilicon nanoparticles [14]. The authors claimed that polysilicon nanoparticles are suitable agents for enhancing oil recovery. They also suggested that the concentration of the nanoparticles plays an important role during the oil recovery process. Similarly, Roustaei et al. demonstrated that SNPs are effective enhanced oil recovery agents for light and intermediate oil reservoirs [25]. The results showed that the utilization of SNPs transformed the rock wettability and efficiently increased the oil recovery from light oil reservoirs.

Two classic mechanisms involved in oil recovery using nanofluids have been reported: (1) nanofluids decrease the interfacial tension between the water and the oil phase and (2) nanofluids can alter the rock wettability [3, 20, 26, 34]. Recently, a new mechanism of oil displacement from a solid substrate using nanoparticles has been proposed [15, 33]. It is based on the ability of the nanoparticles to form two-dimensional layered structures in the confines of the three-phase (solid-oil-aqueous phase) contact line. This phenomenon induces a pressure gradient within the nanofluid, which is responsible for spreading the nanofluid over the solid surface, detaching the oil.

The three mechanisms mentioned above highly depend on the interaction between the NPs and porous media [32, 34]. It has been reported that the interaction between these two systems is affected by some parameters such as, shape and size of nanoparticles, nanoparticle concentration, particle charge, polydispersity and colloidal stability [15, 32-34]. There are several studies reporting the influence of size and concentration of SNPs, colloidal stability of SNPs, types of silica nanoparticles and temperature on oil recovery [30-32].
For example, Onyekonwu et al. used three different polysilicon nanoparticles (lipohydrophilic, hydrolipophobic and medium-wet nanoparticles) to evaluate their impact on oil recovery [20]. They concluded that these three types of nanoparticles are suitable oil recovery agents. Similarly, Ju et al. conducted a study using untreated polysilicon nanoparticle, lipophobic and hydrophilic polysilicon nanoparticle to evaluate the wettability alteration due to the adsorption of NPs on porous walls of sandstone [13]. Their results revealed that the nanoparticles were able to improve the relative permeability of water in porous media, thus, enhancing oil recovery. The effect of the size of SNPs on oil recovery was reported by Hendraningrat et al. [32]. The results indicated that oil recovery is inversely proportional to SNP size. On the other hand, Miranda et al. reported that high temperature and salinity affected the diffusion coefficient of SNPs and the interfacial tension between the SNPs and the bulk [18].

Other groups have studied the effect of concentration and colloidal stability of SNPs [11,25]. These studies confirmed that these parameters are critical for an effective oil recovery. Despite all these reported results, to our knowledge, no study has been undertaken experimentally to investigate the effect of surface charge of SNPs on oil recovery. We hypothesize that the surface charge of SNPs could impact oil recovery, since this parameter could significantly alter the wettability of the reservoir rocks. To prove this hypothesis, SNPs with different charge profiles: negative (Neg-SNPs; $-33.45 \pm 2.75$ ), positive (AP-SNPs; $+42.25 \pm 1.90$ ) and slightly negative (MeO-PEG-SNPs; $-12.20 \pm 0.42$ ) were synthesized and characterized using transmission electron microscopy (TEM), FT-IR, dynamic light scattering (DLS) and $\zeta$-potential. In addition, the effect of the surface charge of SNPs on wettability alteration and oil recovery was evaluated by contact angle estimation and spontaneous imbibition experiments. The results demonstrated that SNPs with different surface charge profiles were able to influence the rock wettability and increased the oil recovery. At a concentration of $100 \mathrm{mg} / \mathrm{L}, \mathrm{MeO}-\mathrm{PEG}-\mathrm{SNPs}$ had the best performance in changing the rock wettability from an oil wet to a water wet state as determined by contact angle estimation. These results are in agreement with imbibition experiments which demonstrated that these NPs showed the higher oil displacement. However, at a higher concentration $(200 \mathrm{mg} / \mathrm{L})$, the effectiveness of Neg-SNPs and AP-SNPs increased as demonstrated by contact angle and imbibition experiments, with Neg-SNPs showing the best performance. These results offer an understanding of the influence of surface charge on oil recovery, which may provide valuable information to maximize oil recovery using nanofluids. 


\section{Experimental details}

\section{Materials}

Tetraethyl orthosilicate (TEOS 98\%), ammonium hydroxide solution (31.5\%), methoxy polyethylene glycol (MeOPEG, 2K), 3-aminopropyl (AP) triethoxysilane (APTES), 3-(triethoxysilyl)propyl isocyanate and absolute ethanol (95\%) were obtained from Sigma-Aldrich (St Louis, MO, USA). Crude oil was obtained from Texas Raw Crude (Midland, TX, USA). Berea sandstone core plugs were purchased from Berea Sandstone Petroleum Cores (Vermilion, $\mathrm{OH}, \mathrm{USA})$. All the reagents and solvents were used as received without further purification.

\section{Synthesis and characterization of silica nanoparticles with different charge profiles}

For the synthesis of silica nanoparticles with different charge profiles, a multistep procedure was carried out by following the Stöber process using TEOS as a silica source and aqueous ammonium hydroxide as a catalyst [22]. First, $50 \mathrm{~mL}$ of a solution containing ammonium hydroxide $(12 \mathrm{mmol})$, and deionized water $(60 \mathrm{mmol})$ in ethanol was stirred for $5 \mathrm{~min}$. Then, $10 \mathrm{~mL}$ of a mixture of TEOS (24 mmol) and ethanol was added to the previous solution and stirred overnight at room temperature. Afterward, SNPs were recovered by centrifugation at high speed, washed twice with ethanol, resuspended in ethanol and stored in the same solvent. The silanol groups present on the surface of the SNPs after this synthetic pathway provided negatively charged nanoparticles (Neg-SNPs; $-33.45 \pm 2.75)$. The second step was the functionalization of the Neg-SNPs with various functional groups to render slightly negative and positive surface charges. Methoxy polyethylene glycol $\left(\mathrm{MeO}-\mathrm{PEG}_{2 \mathrm{~K}}\right)$ was used to modify the surface of the Neg-SNPs to a slightly negatively charged surface (MeO-PEG-SNPs; $-12.20 \pm 0.42$ ). $\mathrm{MeO}-\mathrm{PEG}_{2 \mathrm{~K}}$ has a near neutral $\zeta$-potential and has the ability to neutralize the negative charged surface provided by silanol groups. This functionalization step involved the modification of one end of the MeO- $\mathrm{PEG}_{2 \mathrm{~K}}$ chain to obtain a $\mathrm{MeO}-\mathrm{PEG}_{2 \mathrm{~K}}$ silane derivative for binding on the surface of silica nanoparticles. To synthesize the $\mathrm{MeO}-\mathrm{PEG}_{2 \mathrm{~K}}$-silane, $302.2 \mathrm{mg}(0.15 \mathrm{mmol})$ of dehydrated $\mathrm{MeO}-\mathrm{PEG}_{2 \mathrm{~K}}$ were dissolved in $10 \mathrm{~mL}$ of dichloromethane. Then, $40.4 \mu \mathrm{L}$ of 3-(triethoxysilyl)propyl isocyanate was added and the final solution was stirred overnight at $0{ }^{\circ} \mathrm{C}$. After this period of time, the final product was concentrated by rotary evaporation, dried in a vacuum and characterized using ${ }^{1} \mathrm{H}-\mathrm{NMR}$ and ${ }^{13} \mathrm{C}$-NMR. Next, $100 \mathrm{mg}$ of Neg-SNPs were redispersed in $30 \mathrm{ml}$ of ethanol followed by the addition of $160 \mathrm{mg}$ of the $\mathrm{MeO}-\mathrm{PEG}_{2 \mathrm{~K}}$-silane derivative. The final solution was refluxed overnight at $90{ }^{\circ} \mathrm{C}$. MeO-PEG-SNPs were washed three times with ethanol and stored in the same solvent.

The positively charged nanoparticles (AP-SNPs; $+42.25 \pm 1.9$ ) were obtained grafting (3-aminopropyl) triethoxysilane (APTES) onto the surface of Neg-SNPs by ethanolic refluxing. To this, $100 \mathrm{mg}$ of Neg-SNPs were redispersed in $30 \mathrm{~mL}$ of ethanol. Then, $0.6 \mathrm{mmol}$ of APTES was added to the nanoparticle solution and stirred overnight at $90{ }^{\circ} \mathrm{C}$ under refluxing conditions. AP-SNPs were washed three times with ethanol, resuspended in ethanol and stored in the same solvent.

The particle size and morphology of the negatively, slightly negatively and positively charged nanoparticles were determined using a JEOL JEM 2100 LaB6 Transmission Electron Microscope (TEM). The hydrodynamic diameter and the $\zeta$-potential of the nanoparticles in water were determined using a particle-size analyzer (Zetasizer Nano; Malvern Instruments, Malvern, UK). FT-IR analysis were performed to further confirm the successful surface modification of the silica materials (Perkin-Elmer 100 IR spectrophotometer).

\section{Contact angle estimation}

Berea sandstone core plugs were cut into small pieces and aged in crude oil for 7 days at $55{ }^{\circ} \mathrm{C}$ to alter the wetting tendency of their surface to an oily-wet [9]. After this period of time, sandstone cores were removed from the crude oil and placed in an oven until dried. The aged sandstone core pieces were immersed into an aqueous solution of $0.3 \% \mathrm{NaCl}$ containing different concentrations of silica materials (100 or $200 \mathrm{mg} / \mathrm{L}$ ) for $24 \mathrm{~h}$. Afterward, the samples were removed and placed in an oven until dried. Then, a droplet of water was added onto the surface of silica materials-treated sandstone and the angle between the solid surface and the tangent to the drop profile at the drop edge (contact angle) was measured by visual estimation [9]. As a control, contact angle estimation in untreated aged sandstone was also performed.

\section{Imbibition experiments}

For spontaneous imbibition experiments, Berea sandstone core plugs were cut into adequate pieces and aged in crude oil to alter the wetting tendency of their surface to an oilywet as established previously [9]. After this process, the mass of oil adsorbed by the sandstone core was determined by the difference between the mass of the dry core and the mass of the oil-treated core $\left(M_{\text {oil }}=M_{\text {oil treated core }}-M_{\text {dry core }}\right)$. The volume of the oil present in the sandstone cores was calculated using the density of the crude oil $(0.7 \mathrm{~g} / \mathrm{mL})$. Then, 
the aged sandstone core pieces were immersed into a glass jar containing a $0.3 \% \mathrm{NaCl}$ aqueous solution and different concentrations of silica materials ( 100 or $200 \mathrm{mg} / \mathrm{L}$ ). The oil that was expelled from the Berea sandstone as the nanoparticle solution imbibed was removed from the top of the glass jar using a glass pipette. The samples were centrifuged at high speed to remove the test solution and the amount of oil displaced by the solution of silica materials (expressed as a percentage of the original amount of oil in the sandstone cores) was estimated as a function of time. The imbibition studies were performed also in untreated cores.

\section{Results and discussion}

\section{Characterization of silica nanoparticles with different charge profiles}

The surface charge of nanoparticles may play an important role in oil recovery. To evaluate the effect of the surface charge of SNPs on oil recovery, SNPs with different charges (negative, positive and slightly negative) were prepared following a multistep procedure. First, the Stöber process was used to synthesize SNPs using TEOS as the silica source and ammonium hydroxide as a catalyst. The final material obtained from this synthetic pathway exhibited a negative surface charge due to the silanol groups present on its surface (Neg-SNPs). Next, the slightly negatively and positively charged nanoparticles were obtained by modifying the surface of Neg-SNPs with $\mathrm{MeO}-\mathrm{PEG}_{2 \mathrm{~K}}$ silane and APTES, respectively. Before grafting the $\mathrm{MeO}-\mathrm{PEG}_{2 \mathrm{~K}}$ silane onto the surface of Neg-SNPs, its successful synthesis in situ was confirmed by NMR (supplementary material, Fig. S1): ${ }^{1} \mathrm{H}-\mathrm{NMR}(300 \mathrm{MHz}): \delta(\mathrm{t}, 2 \mathrm{H}, 0.63),(\mathrm{t}, 9 \mathrm{H}, 1.20),(\mathrm{m}, 2 \mathrm{H}$, 1.68), (t, $2 \mathrm{H}, 3.37)$, (s, 3H, 3.49), (m, 164H, 3.50-3.65), (q, 6H, 3.76); ${ }^{13} \mathrm{C}-\mathrm{NMR}(300 \mathrm{MHz}): \delta 7.76\left(\mathrm{CH}_{2}\right), 18.26\left(\mathrm{CH}_{3}\right)$, $25.14\left(\mathrm{CH}_{2}\right), 45.45\left(\mathrm{CH}_{2}\right), 58.46\left(\mathrm{O}-\mathrm{CH}_{2}\right), 61.67\left(\mathrm{O}-\mathrm{CH}_{3}\right)$, 70.4-72.6 (O- $\mathrm{CH}_{2}$ PEG), $156.48(\mathrm{C}=\mathrm{O})$.

Finally, the structural properties of the nanoparticles were determined by dynamic light scattering, $\zeta$-potential and TEM. According to TEM images (Fig. 1), the silica materials with different charge profiles were fabricated with a diameter of $59.6 \pm 5.8 \mathrm{~nm}$. The hydrodynamic diameter of Neg-SNPs was $78.8 \pm 19.0 \mathrm{~nm}$. Neg-SNPs modified with



Fig. 1 TEM images of a Neg-SNPs, b AP-SNPs, and c MeO-PEG-SNPs. The diameter of the synthesized nanoparticles was around $59.6 \pm 5.8 \mathrm{~nm}$ 
APTES showed an increase in the hydrodynamic diameter $(114.0 \pm 16.6 \mathrm{~nm})$. When $\mathrm{MeO}-\mathrm{PEG}_{2 \mathrm{~K}}$ was grafted on the nanoparticles, the hydrodynamic diameter increased considerably to $254.6 \pm 181.0 \mathrm{~nm}$. We believe that this increase in hydrodynamic diameter may be due to agglomeration of the nanoparticles as seen in the TEM images. The $\zeta$-potential results demonstrated that SNPs were successfully modified to afford different charge profiles as shown in Table 1 . NegSNPs showed a $\zeta$-potential of $-33.45 \pm 2.75 \mathrm{mV}$ indicating the presence of silanol groups on their surface. When SNPs were covered with MeO-PEG ${ }_{2 \mathrm{~K}}$ (MeO-PEG-SNPs), the $\zeta$-potential decreased to $-12.20 \pm 0.42 \mathrm{mV}$, which indicates that the silanol groups were successfully neutralized by the polymer. On the other hand, SNPs modified with APTES (AP-SNPs) showed a $\zeta$-potential of $+42.25 \pm 1.90 \mathrm{mV}$, which demonstrates the presence of amine groups on the surface of the nanoparticles. To further confirm the modification of the synthesized nanoparticles, FT-IR analysis

Table 1 Structural properties of the synthesized nanoparticles

\begin{tabular}{lcc}
\hline Material & $\begin{array}{l}\text { Hydrodynamic diam- } \\
\text { eter }(\mathrm{nm})\end{array}$ & $\zeta$-potential $(\mathrm{mV})$ \\
\hline Neg-SNPs & $78.8 \pm 19.0$ & $-33.45 \pm 2.75$ \\
AP-SNPs & $114.0 \pm 16.6$ & $+42.25 \pm 1.90$ \\
MeO-PEG-SNPs & $254.6 \pm 181.0$ & $-12.20 \pm 0.42$ \\
\hline
\end{tabular}

were performed. The FT-IR spectrum of Neg-SNPs (Fig. 2) showed the characteristics bands of $\mathrm{Si}-\mathrm{O}-\mathrm{Si}$ asymmetric vibration and $\mathrm{Si}-\mathrm{OH}$ asymmetric stretching vibration at 1048 and $948 \mathrm{~cm}^{-1}$, respectively [8]. After modification of Neg-SNPs with APTES (AP-SNPs), a new peak at $1556 \mathrm{~cm}^{-1}$ due to $\mathrm{N}-\mathrm{H}$ bending vibration of amine groups is observed [4], which demonstrates that the amino groups were grafted onto the surface of Neg-SNPs. When the SNPs were modified with $\mathrm{MeO}-\mathrm{PEG}_{2 \mathrm{~K}}$ (MeO-PEG-SNPs) it was observed the stretching vibrations at 1690 and $2982 \mathrm{~cm}^{-1}$ corresponding to the carbonyl of the carbamate group and the $\mathrm{C}-\mathrm{H}$ of the PEG chain, respectively. These results confirm the successful fabrication of SNPs with different charge profiles.

\section{Effect of silica nanoparticles with different charge profiles on the wettability of Berea sandstone cores and oil displacement}

Wettability alteration of rock reservoirs plays an important role in oil recovery and it is used to determine the efficacy of nanomaterials during the oil recovery process [34]. To investigate the effect of the surface charge of SNPs on oil recovery, two techniques were performed: contact angle estimation and spontaneous imbibition experiments.

For contact angle estimation, the sandstone cores were submerged in crude oil for 7 days to change the wettability

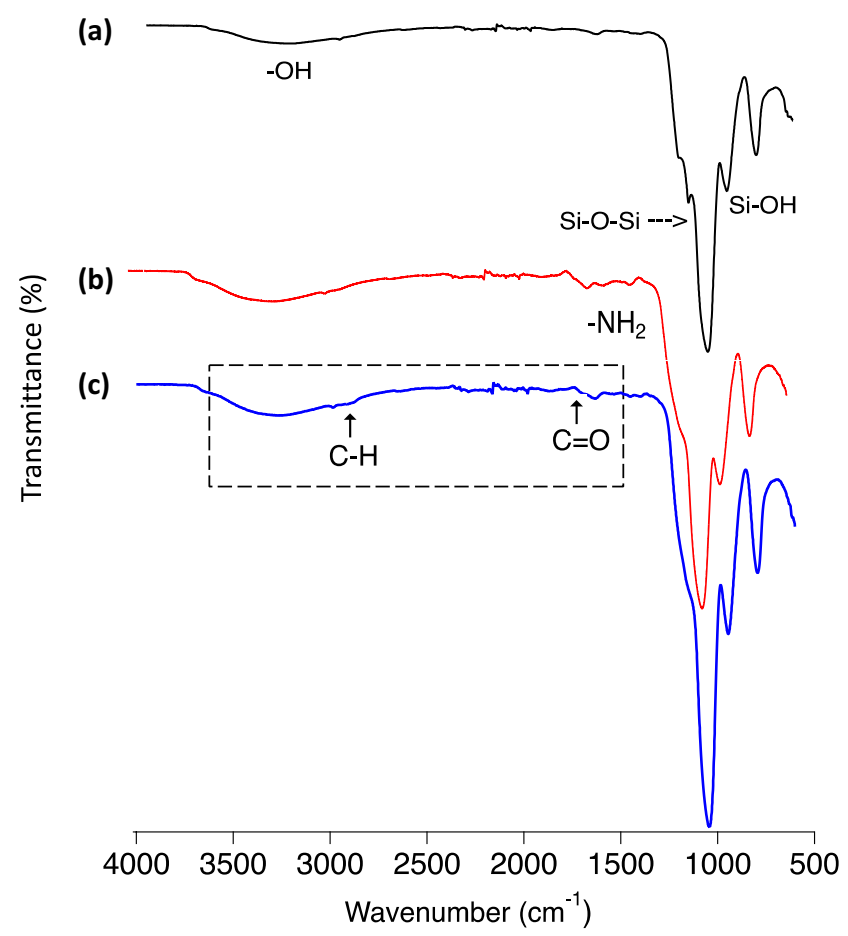

(d)
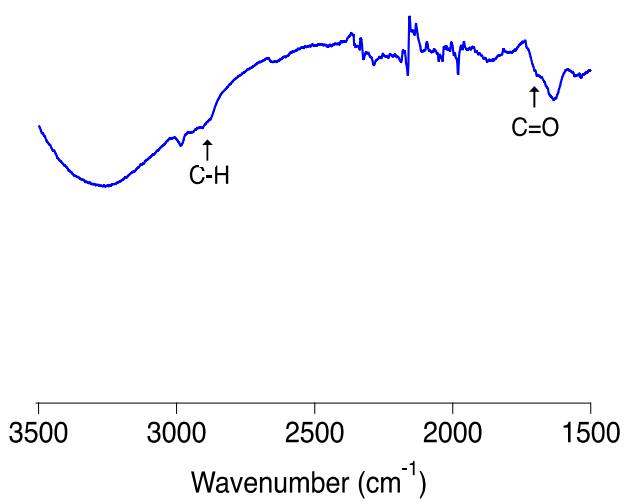

Fig. 2 FT-IR spectra of a Neg-SNPs, b AP-SNPs, $\mathbf{c}$ MeO-PEG-SNPs, and $\mathbf{d}$ close-up on the carbonyl group and the C-H of the PEG chain present on MeO-PEG-SNPs 
of the rock to an oil-wet. Then, the crude oil-treated sandstone cores were exposed to variant concentrations of nanoparticles for $24 \mathrm{~h}$ and the contact angle was determined by visual estimation after adding a droplet of water on the surface of the treated sandstone cores.

The results demonstrated that the three types of silica materials were able to alter the wettability of oil-treated sandstone cores at different extents depending on the concentration (Fig. 3). Untreated sandstone cores showed an estimated contact angle of $106.32^{\circ}$, which confirms their oilwet nature after the treatment with the crude oil. After exposure to silica materials with different charges, the wettability of oil-treated sandstone cores was modified. At $100 \mathrm{mg} / \mathrm{L}$, MeO-PEG-SNPs were able to change the contact angle to $74^{\circ}$. Neg-SNPs altered the contact angle to $94.54^{\circ}$. On the other hand, core plugs treated with AP-SNPs changed the contact angle to $100.24^{\circ}$. These results demonstrated that at a low concentration the nanoparticles with slightly negative charge (MeO-PEG-SNPs) were more effective in modifying

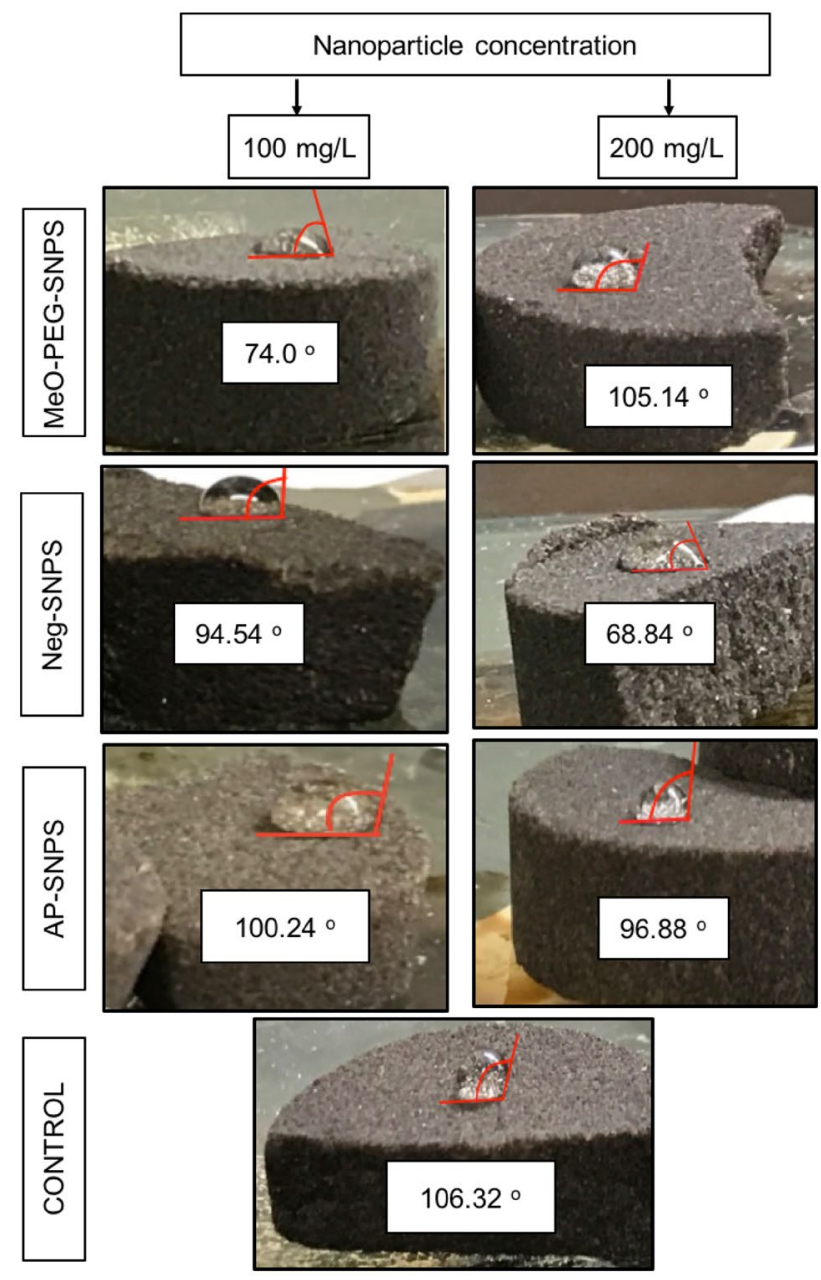

Fig. 3 Contact angle estimation after treatment with silica nanoparticles at different concentrations the wettability of the sandstone cores. However, when the concentration increased to $200 \mathrm{mg} / \mathrm{L}, \mathrm{MeO}-\mathrm{PEG}-\mathrm{SNP}$ treated sandstone cores showed a contact angle of $105.14^{\circ}$, indicating that at this concentration, the wettability of the oil-treated sandstone cores was slightly altered. On the other hand, the treatment of core plugs with Neg-SNPs and AP-SNPs induced a change in contact angle of $68.84^{\circ}$ and $96.88^{\circ}$, respectively. These results indicated that at a higher concentration, nanoparticles with a negative surface charge (Neg-SNPs) were the most effective in altering the wettability of the rocks from an oil-wet to a water-wetting condition. AP-SNPs increased their performance as the concentration increased. The decrease in effectivity of MeO-PEG-SNPs at $200 \mathrm{mg} / \mathrm{L}$ may be because at higher concentrations they agglomerate and precipitate. This may be one of the possible reasons that inhibits the efficient contact of the nanoparticles with the porous media [9].

For imbibition experiments, oil-treated Berea sandstone cores were exposed to different concentrations of silica materials ( 100 or $200 \mathrm{mg} / \mathrm{L}$ ) and the amount of oil recovered was determined as a function of time. Results demonstrated that the non-treated sandstone cores showed the lowest crude oil displacement performance. However, when sandstone cores were exposed to silica materials, the crude oil displacement performance increased showing different
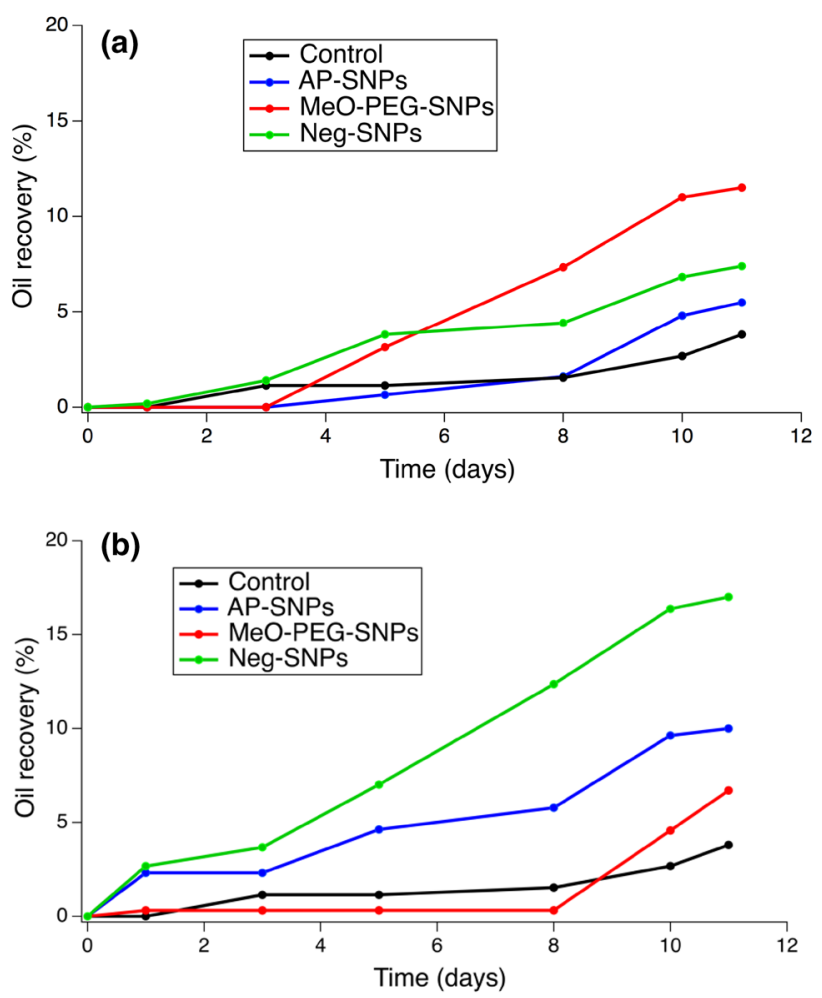

Fig. 4 Spontaneous imbibition curves for sandstone cores exposed to: a silica nanoparticles with different charge profiles at $100 \mathrm{mg} / \mathrm{L}$. b Silica nanoparticles with different charge profiles at $200 \mathrm{mg} / \mathrm{L}$ 
trends (Fig. 4). At a low concentration (100 mg/L), MeOPEG-SNPs showed the highest oil recovery as a function of time. After 11 days of exposure, the percentage of oil displacement was $11.5 \%$. At this condition, Neg-SNPs demonstrated to be more effective in removing oil from sandstone cores than AP-SNPs (7.4 and 5.5\% of oil recovery, respectively). Contrary, when the concentration of the nanoparticles increased to $200 \mathrm{mg} / \mathrm{L}$; MeO-PEG-SNPs showed the lowest effectivity in removing oil from sandstone cores. After 11 days of treatment, the percentage of oil decreased to $6.7 \%$. On the other hand, negatively charged nanoparticles (Neg-SNPs) demonstrated to be the most effective for this particular application with a percentage of oil recovery of $17 \%$. AP-SNPs were more effective at a higher concentration that at a low concentration as shown by the increase in percentage of oil recovery from $5.5 \%(100 \mathrm{mg} / \mathrm{L})$ to $10 \%$ $(200 \mathrm{mg} / \mathrm{L})$. These results indicated that slightly negatively charged nanoparticles are more effective in oil recovery at low concentrations. Nevertheless, the performance of negatively and positively charged nanoparticles improved by increasing nanoparticles concentration. The imbibition experiments were in agreement with contact angle estimation results. It is known that water-wet nature increases the spontaneous imbibition process. Consequently, $\mathrm{MeO}-$ PEG-SNP-treated sandstone cores which showed the most strongly water-wet nature at a low concentration; exhibited the faster spontaneous imbibition process. In contrast, those samples that showed the most strongly water-wet nature at a higher concentration (Neg-SNPs treated sandstone and APSNPs-treated sandstone cores), also had the highest performance in oil displacement. Taking together these results, demonstrated that the surface charge of SNPs impacted oil recovery in different extent. We believe that the decrease in efficiency of slightly negatively charged nanoparticles at a higher concentration $(200 \mathrm{mg} / \mathrm{L})$ is due to precipitation of MeO-PEG-SNPs, which could affect the interface between the oil and water phases.

\section{Conclusion}

Our work demonstrated that silica nanoparticles with different surface charges were capable of altering the wettability of sandstone cores as determined by contact angle estimation. Additionally, these materials impacted the oil recovery process in different extents. While MeO-PEGSNPs (slightly negatively charged nanoparticles) showed the highest oil recovery at a low concentration $(100 \mathrm{mg} / \mathrm{L})$, at a higher concentration (200 mg/L), Neg-SNPs demonstrated to be the most effective in recovering oil from sandstone cores. Moreover, the concentration plays an important role in evaluating the impact of the surface charge on oil recovery. As the concentration increased, Neg-SNPs and AP-SNPs improved the wettability and the oil recovery process. On the other hand, the effectiveness of MeO-PEG-SNPs decreased as the concentration increased. Overall, the results obtained from this study could not only give a deeper insight of the interaction between nanofluids and porous media, but could also provide additional information about the optimal structural characteristics that SNPs should meet to maximize oil recovery.

Acknowledgements We thank the Inter American University of Puerto Rico (Seed Grant funding) for supporting this research work.

\section{Compliance with ethical standards}

Conflict of interest On behalf of all authors, the corresponding author states that there is no conflict of interest.

Open Access This article is distributed under the terms of the Creative Commons Attribution 4.0 International License (http://creativeco mmons.org/licenses/by/4.0/), which permits unrestricted use, distribution, and reproduction in any medium, provided you give appropriate credit to the original author(s) and the source, provide a link to the Creative Commons license, and indicate if changes were made.

\section{References}

1. Al-Anssari, S., Barifcani, A., Wang, S., Maxim, L., Iglauer, S.: Wettability alteration of oil-wet carbonate by silica nanofluid. J. Colloid Interface Sci. 461, 435-442 (2016). https://doi. org/10.1016/j.jcis.2015.09.051

2. Anderson, W.G.: Wettability literature survey-part 6: the effects of wettability on waterflooding. J. Pet. Technol. 39, 1605-1622 (1987). https://doi.org/10.2118/16471-PA

3. Aoudia, M., Al-Maamari, R.S., Nabipour, M., Al-Bemani, A.S., Ayatollahi, S.: Laboratory study of alkyl ether sulfonates for improved oil recovery in high-salinity carbonate reservoirs: a case study. Energy Fuels 24, 3655-3660 (2010). https://doi. org/10.1021/ef100266p

4. Azarshin, S., Moghadasi, J., A Aboosadi, Z.: Surface functionalization of silica nanoparticles to improve the performance of water flooding in oil wet reservoirs. Energy Explor. Exploit. 35, 685-697 (2017). https://doi.org/10.1177/0144598717716281

5. Cheraghian, G., Hendraningrat, L.: A review on applications of nanotechnology in the enhanced oil recovery part B: effects of nanoparticles on flooding. Int. Nano Lett. 6, 1-10 (2015). https:// doi.org/10.1007/s40089-015-0170-7

6. Ehtesabi, H., Ahadian, M.M., Taghikhani, V., Ghazanfari, M.H.: Enhanced heavy oil recovery in sandstone cores using $\mathrm{TiO}_{2}$ nanofluids. Energy Fuels 28, 423-430 (2014). https://doi.org/10.1021/ ef $401338 \mathrm{c}$

7. Esfandyari Bayat, A., Junin, R., Samsuri, A., Piroozian, A., Hokmabadi, M.: Impact of metal oxide nanoparticles on enhanced oil recovery from limestone media at several temperatures. Energy Fuels 28, 6255-6266 (2014). https://doi.org/10.1021/ef5013616

8. Feifel, S.C., Lisdat, F.: Silica nanoparticles for the layer-by-layer assembly of fully electro-active cytochrome $c$ multilayers. J. Nanobiotechnol. 9, 59 (2011). https://doi.org/10.1186/1477-3155-9-59

9. Giraldo, J., Benjumea, P., Lopera, S., Cortés, F.B., Ruiz, M.A.: Wettability alteration of sandstone cores by alumina-based 
nanofluids. Energy Fuels 27, 3659-3665 (2013). https://doi. org/10.1021/ef4002956

10. Hendraningrat, L., Li, S., Torsæter, O.: A coreflood investigation of nanofluid enhanced oil recovery. J. Pet. Sci. Eng. 111, 128-138 (2013). https://doi.org/10.1016/j.petrol.2013.07.003

11. Hendraningrat, L., Torsæter, O.: Metal oxide-based nanoparticles: revealing their potential to enhance oil recovery in different wettability systems. Appl. Nanosci. 5, 181-199 (2014). https://doi. org/10.1007/s13204-014-0305-6

12. Holzinger, M., Le Goff, A., Cosnier, S.: Nanomaterials for biosensing applications: a review. Front. Chem. 2, 63 (2014). https ://doi.org/10.3389/fchem.2014.00063

13. Ju, B., Dai, S., Luan, Z., Zhu, T., Su, X., Qiu, X.: A study of wettability and permeability change caused by adsorption of nanometer structured polysilicon on the surface of porous media. In: Paper presented at the SPE Asia Pacific Oil and Gas Conference and Exhibition, Melbourne, Australia, 2002/1/1 (2002)

14. Ju, B., Fan, T., Ma, M.: Enhanced oil recovery by flooding with hydrophilic nanoparticles. China Particuol. 4, 41-46 (2006). https ://doi.org/10.1016/S1672-2515(07)60232-2

15. Kao, R.L., Wasan, D.T., Nikolov, A.D., Edwards, D.A.: Mechanisms of oil removal from a solid surface in the presence of anionic micellar solutions. Colloids Surf. 34, 389-398 (1988). https ://doi.org/10.1016/0166-6622(88)80163-X

16. Karimi, A., Fakhroueian, Z., Bahramian, A., Pour Khiabani, N., Darabad, J.B., Azin, R., Arya, S.: Wettability alteration in carbonates using zirconium oxide nanofluids: EOR implications. Energy Fuels 26, 1028-1036 (2012). https://doi.org/10.1021/ef201475u

17. Levitt, D., Pope, G.A.: Selection and screening of polymers for enhanced-oil recovery. In: Paper presented at the SPE Symposium on Improved Oil Recovery, Tulsa, Oklahoma, USA, 20-23 April (2008)

18. Miranda, C.R., Lara, L.S.D., Tonetto, B.C.: Stability and mobility of functionalized silica nanoparticles for enhanced oil recovery applications. In: Paper presented at the SPE International Oilfield Nanotechnology Conference and Exhibition, Noordwijk, The Netherlands, 12-14 June (2012)

19. Nazari Moghaddam, R., Bahramian, A., Fakhroueian, Z., Karimi, A., Arya, S.: Comparative study of using nanoparticles for enhanced oil recovery: wettability alteration of carbonate rocks. Energy Fuels 29, 2111-2119 (2015). https://doi.org/10.1021/ ef5024719

20. Onyekonwu, M.O., Ogolo, N.A.: Investigating the use of nanoparticles in enhancing oil recovery. In: Paper presented at the Nigeria Annual International Conference and Exhibition, Tinapa-Calabar, Nigeria, 31 July-7 August (2010)

21. Ponnapati, R., Karazincir, O., Dao, E., Ng, R., Mohanty, K.K., Krishnamoorti, R.: Polymer-functionalized nanoparticles for improving waterflood sweep efficiency: characterization and transport properties. Ind. Eng. Chem. Res. 50, 13030-13036 (2011). https://doi.org/10.1021/ie2019257

22. Qiao, Z.-A., Zhang, L., Guo, M., Liu, Y., Huo, Q.: Synthesis of mesoporous silica nanoparticles via controlled hydrolysis and condensation of silicon alkoxide. Chem. Mater. 21, 3823-3829 (2009). https://doi.org/10.1021/cm901335k

23. Rahman, I.A., Padavettan, V.: Synthesis of silica nanoparticles by sol-gel: size-dependent properties, surface modification, and applications in silica-polymer nanocomposites-a review. J. Nanomater. 2012, 15 (2012). https://doi.org/10.1155/2012/132424

24. Roustaei, A., Bagherzadeh, H.: Experimental investigation of $\mathrm{SiO}_{2}$ nanoparticles on enhanced oil recovery of carbonate reservoirs. J. Pet. Explor. Prod. Technol. 5, 27-33 (2014). https://doi. org/10.1007/s13202-014-0120-3

25. Roustaei, A., Saffarzadeh, S., Mohammadi, M.: An evaluation of modified silica nanoparticles' efficiency in enhancing oil recovery of light and intermediate oil reservoirs. Egypt. J. Pet. 22, 427-433 (2013). https://doi.org/10.1016/j.ejpe.2013.06.010

26. Shahrabadi, A., Bagherzadeh, H., Roostaie, A., Golghanddashti, H.: Experimental investigation of HLP nanofluid potential to enhance oil recovery: a mechanistic approach. In: Paper presented at the SPE International Oilfield Nanotechnology Conference and Exhibition, 12-14 June, Noordwijk, Netherlands, June 12-14 (2012)

27. Shin, Y., Lee, D., Lee, K., Ahn, K.H., Kim, B.: Surface properties of silica nanoparticles modified with polymers for polymer nanocomposite applications. J. Ind. Eng. Chem. 14, 515-519 (2008). https://doi.org/10.1016/j.jiec.2008.02.002

28. Singh, S.K., Kulkarni, P.P., Dash, D.: Biomedical applications of nanomaterials: an overview. In: Bio-nanotechnology. Blackwell Publishing Ltd., pp. 1-32. (2013). https://doi.org/10.1002/97811 18451915.ch1

29. Tabary, R., Bazin, B., Douarche, F., Moreau, P., OukhemanouDestremaut, F.: Surfactant flooding in challenging conditions: towards hard brines and high temperatures. In: Paper presented at the SPE Middle East Oil and Gas Show and Conference, Manama, Bahrain, 10-13 March (2010)

30. Torsaeter, O., Li, S., Hendraningrat, L.: Enhancing oil recovery of low-permeability berea sandstone through optimised nanofluids concentration. In: Paper presented at the SPE Enhanced Oil Recovery Conference, Kuala Lumpur, Malaysia, 2013/7/2 (2013)

31. Torsater, O., Li, S., Hendraningrat, L.: A coreflood investigation of nanofluid enhanced oil recovery in low-medium permeability berea sandstone. In: Paper presented at the SPE International Symposium on Oilfield Chemistry, The Woodlands, Texas, USA, 2013/4/8 (2013a)

32. Torsater, O., Li, S., Hendraningrat, L.: Effect of some parameters influencing enhanced oil recovery process using silica nanoparticles: an experimental investigation. In: Paper presented at the SPE Reservoir Characterization and Simulation Conference and Exhibition, Abu Dhabi, UAE, 16-18 September (2013b)

33. Wasan, D.T., Nikolov, A.D.: Spreading of nanofluids on solids. Nature 423, 156-159 (2003). http://www.nature.com/nature/journ al/v423/n6936/suppinfo/nature01591_S1.html

34. Zhang, H., Nikolov, A., Wasan, D.: Enhanced oil recovery (EOR) using nanoparticle dispersions: underlying mechanism and imbibition experiments. Energy Fuels 28, 3002-3009 (2014). https:// doi.org/10.1021/ef500272r

Publisher's Note Springer Nature remains neutral with regard to jurisdictional claims in published maps and institutional affiliations. 